\section{PHASE 1B/2 KEYNOTE-365 COHORT I: PLATINUM- CONTAINING CHEMOTHERAPY ALONE OR IN COMBINATION WITH PEMBROLIZUMAB FOR TREATMENT-EMERGENT NEUROENDOCRINE PROSTATE CARCINOMA}

${ }^{1}$ Johann De Bono*, ${ }^{2}$ Neal Shore, ${ }^{3}$ Gero Kramer, ${ }^{4}$ Anthony Joshua, ${ }^{5}$ Xin Tong Li, ${ }^{5}$ Christian Poehlein, ${ }^{5}$ Charles Schloss, ${ }^{6}$ Evan Yu. ${ }^{1}$ The Royal Marsden NHS Foundation Trust, London, UK; ${ }^{2}$ Carolina Urologic Research Center, Myrtle Beach, SC, USA; ${ }^{3}$ Medical University of Vienna, Vienna, Austria; ${ }^{4}$ Saint Vincent's Hospital Sydney, Sydney, Australia; ${ }^{5}$ Merck and Co., Inc., Kenilworth, NJ, USA; ${ }^{6}$ University of Washington, Seattle, WA, USA

Background Treatment-emergent neuroendocrine prostate carcinoma (t-NE) can occur de novo or after diagnosis of prostate adenocarcinoma. Treatment often includes platinum-containing chemotherapy because of t-NE's histologic similarity to small cell lung cancer. The PD-1 inhibitor pembrolizumab has shown promising efficacy and acceptable safety when combined with olaparib, docetaxel, or enzalutamide for treatment of metastatic castration-resistant prostate cancer (mCRPC) in the multicohort phase $1 \mathrm{~b} / 2$ KEYNOTE-365 study (NCT02861573). Cohort I will be used to compare platinumcontaining chemotherapy alone with chemotherapy + pembrolizumab as treatment for $\mathrm{t}-\mathrm{NE}$.

Methods Patients who have t-NE $(\geq 1 \%$ neuroendocrine cells in a recent biopsy specimen confirmed by central histology review); experienced progression within 6 months of starting a next-generation hormonal agent (NHA) for mCRPC or hormone-sensitive prostate cancer and experienced progression within 6 cycles of docetaxel treatment for mCRPC; and have an Eastern Cooperative Oncology Group (ECOG) performance status score of 0 or 1 are eligible. Prior therapy with $\leq 2$ NHAs and 1 other chemotherapy for mCRPC is permitted. Patients will be randomly assigned 1:1 to receive pembrolizumab $200 \mathrm{mg}$ IV on day 1 of each cycle every 3 weeks + carboplatin AUC of 5 IV on day $1+$ etoposide $100 \mathrm{mg} / \mathrm{m} 2$ IV on days 1, 2, and 3 of each 21-day cycle for 4 cycles (arm 1) or the same chemotherapy regimen without pembrolizumab (arm 2); in each arm 40-100 patients will be enrolled. Pembrolizumab treatment will continue up to 2 years until disease progression, unacceptable toxicity, or withdrawal of consent. Patients will be stratified by ECOG performance status score (0 or 1$)$. Computed tomography or magnetic resonance imaging will be performed every 9 weeks through week 54 and every 12 weeks thereafter. Primary end points are safety and tolerability, prostate-specific antigen (PSA) response rate, and objective response rate (ORR) per RECIST v1.1 by blinded independent central review (BICR). Secondary end points are time to PSA progression; ORR and radiographic progressionfree survival (PFS) per PCWG3-modified RECIST v1.1 by BICR; duration of response and disease control rate per RECIST v1.1 by BICR and PCWG3-modified RECIST v1.1 by BICR; and overall survival. End points will be summarized for each arm without formal hypothesis testing.

Acknowledgements Medical writing and/or editorial assistance was provided by Matthew Grzywacz, PhD, of ApotheCom (Yardley, PA, USA). This assistance was funded by Merck Sharp \& Dohme Corp., a subsidiary of Merck \& Co., Inc., Kenilworth, NJ, USA. Funding for this research was provided by Merck Sharp \& Dohme Corp., a subsidiary of Merck \& Co., Inc., Kenilworth, NJ, USA.

Trial Registration ClinicalTrials.gov, NCT02861573

Ethics Approval The study and the protocol were approved by the Institutional Review Board or ethics committee at each site. 